Pacific Journal of Mathematics 


\section{RINGS WHOSE PROPER CYCLIC MODULES ARE QUASI-INJECTIVE}

\section{S. K. Jain, SurJeet Singh, and R. G. Symonds}

A ring $R$ with identity is a right $P C Q I$-ring ( $P C I$-ring) if every cyclic right $R$-module $C \not R$ is quasi-injective (injective). Left $P C Q I$-rings ( $P C I$-rings) are similarly defined. Among others the following results are proved: (1) A right $P C Q I$-ring is either prime or semi-perfect. (2) A nonprime nonlocal ring is a right $P C Q I$-ring iff every cyclic right $R$ module is quasi-injective or $R \cong\left(\begin{array}{ll}D & D \\ 0 & D\end{array}\right)$, where $D$ is a division ring. In particular, a nonprime nonlocal right $P C Q I$ ring is also a left $P C Q I$-ring. (3) A local right $P C Q I$-ring with maximal ideal $M$ is a right valuation ring or $M^{2}=(0)$. (4) A prime local right $P C Q I$-ring is a right valuation domain. (5) A right $P C Q I$-domain is a right Öre-domain. Faith proved (5) for right $P C I$-domains. If $R$ is commutative then some of the main results of Klatt and Levy on pre-self-injective rings follow as a special case of these results.

Since, in a commutative Dedekind domain $D$, for each nonzero ideal $A, D / A$ is a self-injective ring, or equivalently $D / A$ is a quasiinjective $D$-module, every commutative Dedekind domain is a $P C Q I$ ring. An example of a PCQI-ring which is not a Dedekind domain is given in Levy [14]. Commutative PCQI-rings are precisely the pre-self-injective rings characterized by Klatt and Levy [11]. PCIrings have recently been investigated by Faith [4]. Right selfinjective right $P C Q I$-rings are $q c$-rings which have been studied by Ahsan [1] and Koehler [13].

1. Definitions and preliminaries. Throughout all modules are unitary and right unless specified. An $R$-module $X$ is called injective relative to an $R$-module $M$ if for each short exact sequence $0 \rightarrow N \rightarrow$ $M \rightarrow M / N \rightarrow 0$ the sequence $0 \rightarrow \operatorname{Hom}_{R}(M / N, X) \rightarrow \operatorname{Hom}_{R}(M, X) \rightarrow$ $\operatorname{Hom}_{R}(N, X) \rightarrow 0$ is exact. $X$ is called quasi-injective if $X$ is injective relative to itself. Any $R$-module injective relative to all $R$ modules is called injective. Relative projectivity is defined dually.

A ring $R$ is called a right $q$-ring if each of its right ideals is quasi-injective (see Jain, Mohamed, and Singh [9]). For more results, see [7], [8], [13], [15]. Dually, a ring $R$ is called a right $q^{*}$-ring if each cyclic right $R$-module is quasi-projective (see Koehler [12]).

A ring $R$ is right $q c$-ring if each cyclic right $R$-module is quasiinjective (see Ahsan [1]). A well-known result of Osofsky [16] states 
that $R$ is semisimple artinian iff each cyclic $R$-module is injective. Koehler [13] showed that $R$ is a right $q c$-ring iff $R$ is a finite direct sum of rings each of which is semisimple artinian or a rank $o$ duo maximal valuation ring. As a consequence, every $q c$-ring is both a $q$-ring and $q^{*}$-ring.

In this paper the classes of rings initially called $q$-rings, $q^{*}$-rings, and $q c$-rings have been called $Q$-rings, $Q^{*}$-rings, and $Q C$-rings respectively.

Let $J(R)$ denote the radical of a ring $R . \quad R$ is called semiperfect if $R / J(R)$ is semisimple artinian and idempotents modulo $J(R)$ can be lifted to $R$. If $R$ is semiperfect, then there exists a finite maximal family of primitive orthogonal idempotents $\left\{e_{i}\right\}_{1 \leqq i \geqq n}$ such that $R=\bigoplus \sum_{i=1}^{n} e_{i} R$.

$R$ is called a local ring if it has a unique maximal right ideal which must be the radical $J(R)$.

$R$ is a right valuation ring if the set of all right ideals is linearly ordered. $R$ is a maximal valuation ring if every family of pairwise solvable congruences of the form $x \equiv x_{\alpha}\left(\bmod A_{\alpha}\right)$ has a simultaneous solution where $x_{\alpha} \in R$ and each $A_{\alpha}$ is an ideal in $R$. $R$ is called an almost maximal valuation ring if each of its proper homomorphic images is a maximal valuation ring.

A ring is right duo if every right ideal is two-sided. A ring $R$ has rank $O$ if every prime ideal is a maximal ideal. By duo rings or valuation rings, we shall mean both right and left.

\section{General results.}

Sublemma 1. Let $I$ be a right ideal in a ring $R$ such that $R / I \cong R$. Then $R=I \oplus J$, where $J$ is a right ideal, and thus $I=e R, e=e^{2} \in R$.

Proof. $R / I \cong R$ implies $R / I$ is projective, and hence $I$ is a direct summand of $R$.

Proposition 2. Let $R$ be a right PCQI-ring. If $I$ is a right ideal of $R$ such that $R / I \cong R$, then $I$ is contained in every nonzero two-sided ideal of $R$.

Proof. Let $S$ be a nonzero two-sided ideal of $R$. Then $R / S$ is a qc-ring, hence is semiperfect. Let $f: R / I \rightarrow R$ be an isomorphism. Since $1+I$ generates $R / I, \quad \bar{R}=x R$, where $x=f(1+I)$. Then $I=\operatorname{ann} x=\{r \in R \mid x r=0\}$. So there exists $y \in R$ such that $x y=1$. Since $R / S$ is semiperfect, $(x+S)(y+S)=1+S=(y+S)(x+S)$. Then $1-y x \in S$. Let $a \in I$, i.e., $x a=0$. Then $(1-y x) a=a-y x a=a$, hence $a \in S$. So $I \subseteq S$. 
Proposition 3. Let $R$ be a right PCQI-ring. Then either $R$ is a prime ring or $R$ is semiperfect with nil radical.

Proof. Suppose $R$ is not prime, and $P \neq 0$ is a prime ideal. Then $R / P$ is a $q c$-ring, and hence a $q$-ring. So $R / P$ is simple artinian [9]. Thus $P$ is maximal, hence primitive. So the Jacobson radical is nil.

Since $R$ is not prime, there exist nonzero ideals $A, B$ such that $A B=0$. Since $R$ is a right $P C Q I$-ring, $R / A$ and $R / B$ are semiperfect, hence each of them has finitely many prime ideals. Since every prime ideal of $R$ contains $A$ or $B$, it follows that $R$ has finitely many prime ideals as well. Thus $R / J(R)$ is semisimple artinian, and since $J(R)$ is nil, $R$ is semiperfect.

4. Nonlocal semiperfect PCQI-rings. By Proposition 3, all nonprime right $P C Q I$-rings are semiperfect, so the results of this section hold for the class of nonprime nonlocal right PCQI-rings. The case of local right PCQI-rings is discussed in the next section.

Lemma 4. Let $R$ be a semiperfect ring. Then $R / A$ is a proper cyclic right $R$-module, for all nonzero right ideals $A$.

Proof. There exists a positive integer $n$ such that $R$ is a direct sum of $n$ indecomposable right $R$-modules, and $R$ cannot be expressed as a direct sum of more than $n$ right $R$-modules. Now, if $R / A \cong R$, then, by Lemma $1, R=A \oplus B$ and $B \cong R$. So $A=(0)$, proving the lemma.

Let $R$ be a nonlocal semiperfect ring, and let $\left\{e_{i}\right\}_{1 \leqq i \leqq n}$ be a maximal set of primitive orthogonal idempotents in $R$. Then $R=\bigoplus \sum_{r=1}^{n} e_{i} R$ and $n \geqq 2$. Throughout this section, $e_{i}$ 's will denote primitive idempotents. We shall often use a well-known fact that if $A \oplus B$ is a quasi-injective module then any monomorphism $A \rightarrow B$ splits.

Lemma 5. Let $R$ be a semiperfect nonlocal right PCQI-ring. If $\sigma \in \operatorname{Hom}_{R}\left(e_{i} R, e_{j} R\right)$ such that $\sigma \neq 0$, where $i \neq j$, then $\operatorname{ker} \sigma=(0)$.

Proof. Suppose ker $\sigma \neq(0)$, where $0 \neq \sigma \in \operatorname{Hom}_{R}\left(e_{i} R, e_{j} R\right), i \neq j$. Then $R / \operatorname{ker} \sigma \cong \bigoplus \sum_{\substack{k=1 \\ k \neq i}}^{n} e_{k} R \times \operatorname{Im} \sigma$, and $R / \operatorname{ker} \sigma$ is quasi-injective. Since $\operatorname{Im} \sigma \subseteq e_{j} R$, the inclusion map $i: \operatorname{Im} \sigma \rightarrow \bigoplus \sum_{\substack{k=1 \\ k \neq i}}^{n} e_{k} R$ is a monomorphism. Since $R / \operatorname{ker} \sigma$ is quasi-injective, the inclusion map splits. So $\operatorname{Im} \sigma$ is a direct summand of $e_{j} R$, hence $\operatorname{Im} \sigma=e_{j} R$. Since $e_{j} R$ is projective, $\sigma: e_{i} R \rightarrow e_{j} R$ splits. Thus $\operatorname{ker} \sigma=(0)$. 
LEMMA 6. Let $R$ be a semiperfect nonlocal right PCQI-ring with decomposition $\oplus \sum_{i=1}^{n} e_{i} R$, where $n>2$. Then $\operatorname{Hom}_{R}\left(e_{i} R, e_{j} R\right) \neq 0$ iff $e_{i} R \cong e_{j} R$, i.e., $e_{j} R e_{i} \neq 0$ iff $e_{i} R \cong e_{j} R$.

Proof. Let $\sigma \in \operatorname{Hom}_{R}\left(e_{i} R, e_{j} R\right)$ such that $\sigma \neq 0$. By Lemma 5, ker $\sigma=0$. Since $n>2, e_{i} R \oplus e_{j} R \cong R / \bigoplus \sum_{\substack{k=1 \\ k \neq i, j}}^{n} e_{k} R$ is quasi-injective. Then $\sigma$ splits, and $0 \neq \operatorname{Im} \sigma$ is a direct summand of $e_{j} R$. So $\operatorname{Im} \sigma=e_{j} R$, and $\sigma$ is an isomorphism. The converse is trivial.

Proposition 7. Let $R$ be a semiperfect nonlocal right PCQIring with decomposition $R=\oplus \sum_{i=1}^{n} e_{i} R$, where $n>2$. Then $R$ is a qc-ring.

Proof. For each $i, e_{i} R \cong R / \oplus \sum_{\substack{k=1 \\ k \neq i}}^{n} e_{k} R$. So $e_{i} R$ is quasi-injective, for each $i$. Let $A_{i}$ be the sum of all those $e_{i} R$ which are isomorphic to each other. Then $R=\oplus \sum_{i=1}^{p} A_{i}$. We claim that $A_{i}$ is a two-sided ideal of $R$, for each $i$. Clearly $A_{i}$ is a right ideal. Consider $e_{j} R$ such that $e_{j} R \nsubseteq A_{i}$. Define $f: e_{i} R \rightarrow e_{j} R$, where $e_{i} R \cong A_{i}$, by $f\left(e_{i} r\right)=e_{j} x e_{i} r$, for $x \in R$. Then $f \in \operatorname{Hom}_{R}\left(e_{i} R, e_{j} R\right)$. Since $e_{i} R$ and $e_{j} R$ are not isomorphic, $f=0$ by Lemma 6 . So, for $e_{j} R \varsubsetneqq A_{i}$, $e_{j} R A_{i}=0$. So $R A_{i} \subset A_{i}$. Since $A_{i}$ is a finite direct sum of isomorphic quasi-injective right ideals, $A_{i}$ is quasi-injective, hence a $q c$-ring. Thus, by Koehler [13], $R$ is a $q c$-ring.

Proposition 8. Let $R$ be a semiperfect right PCQI-ring such that $R=e_{1} R \oplus e_{2} R$. If $e_{1} R \cong e_{2} R$, then $R$ is a qc-ring.

Proof. Now $e_{1} R \cong e_{2} R$ and $R / e_{2} R \cong R / e_{1} R$, hence $e_{2} R$ and $e_{1} R$ are quasi-injective. Since $e_{1} R \cong e_{2} R, R=e_{1} R \oplus e_{2} R$ is quasi-injective, hence right self-injective. So $R$ is a $q c$-ring.

Proposition 9. Let $R$ be a semiperfect right PCQI-ring such that $R=e_{1} R \oplus e_{2} R$. If $e_{1} R e_{2}=0$ and $e_{2} R e_{1}=0$, then $R$ is a qc-ring.

Proof. If $e_{1} R e_{2}=0$ and $e_{2} R e_{1}=0$, then $e_{1} R$ and $e_{2} R$ are twosided ideals of $R$. Thus $e_{1} R \cong R / e_{2} R$ and $e_{2} R \cong R / e_{1} R$ are $q c$-rings. Then $R=e_{1} R \oplus e_{2} R$ is a $q c$-ring.

Proposition 10. Let $R$ be a semiperfect right PCQI-ring such that $R=e_{1} R \oplus e_{2} R$. If $e_{1} R e_{2} \neq 0$ and $e_{2} R e_{1} \neq 0$, then $R$ is a qc-ring.

Proof. $e_{1} R e_{2} \neq 0$ and $e_{2} R e_{1} \neq 0$ imply that there exist nonzero homomorphisms, hence monomorphisms by Lemma 5 , from $e_{1} R$ to $e_{2} R$ and from $e_{2} R$ to $e_{1} R$. Thus, by Bumby [2], $e_{1} R \cong e_{2} R$, and Proposition 8 yields the result. 
Proposition 11. Let $R=e_{1} R \oplus e_{2} R$ be a semiperfect right $P C Q I$-ring where $e_{1} R \nRightarrow e_{2} R$ and exactly one of $e_{1} R e_{2}$ or $e_{2} R e_{1}$ is zero. Then $R$ is nonprime with nil radical.

Proof. It follows from that the fact that if $e_{1} R e_{2} \neq 0$, then $e_{1} R e_{2}$ is a nilpotent ideal.

THEOREM 12. Let $R$ be a nonlocal right PCQI-ring. Then $R$ is semiperfect iff $R$ is nonprime or simple artinian.

Proof. Necessity follows by Proposition 3, and sufficiency follows from Proposition 7-11 and Koehler's characterization of $q c$-rings [13] (cf. definitions and preliminaries).

THEOREM 13. Let $R$ be a semiperfect nonlocal ring. Then $R$ is a right PCQI-ring iff either (i) $R=\oplus \sum_{i=1}^{n} R_{i}$, where $R_{i}$ is semisimple artinian or a rank o duo maximal valuation ring or (ii) $R=\left(\begin{array}{ll}D & D \\ 0 & D\end{array}\right)$, where $D$ is a division ring.

Proof. Let $R$ be a right PCQI-ring. By Propositions 7-10, $R$ is a qc-ring unless $R=e_{1} R \oplus e_{2} R$, where $e_{1} R$ and $e_{2} R$ are not isomorphic and exactly one of $e_{1} R e_{2}$ or $e_{2} R e_{1}$ is zero, say $e_{1} R e_{2} \neq 0$ and $e_{2} R e_{1}=0$. If $R$ is a $Q C$-ring, we get (i) by Koehler [13]. Otherwise, we have $R \cong\left(\begin{array}{cc}e_{1} R e_{1} & e_{1} R e_{2} \\ 0 & e_{2} R e_{2}\end{array}\right)$. We claim that $e_{1} R e_{1}$ and $e_{2} R e_{2}$ are isomorphic division rings and $M=e_{1} R e_{2}$ is a $(D, D)$-bimodule such that $\operatorname{dim}_{D} M=1=\operatorname{dim} M_{D}$, where $D \cong e_{1} R e_{1} \cong e_{2} R e_{2}$. Clearly $e_{1} R e_{2}$ is nilpotent ideal and since it is nonzero, $R$ is not prime. So, by Proposition 3, the radical $N$ of $R$ is a nil ideal. Thus $e_{2} N_{2}$ is nil. We claim that $e_{2} N e_{2}=0$. Let $e_{2} x e_{2} \in e_{2} N e_{2}$. Define $\sigma: e_{2} R \rightarrow e_{2} R$ by $\sigma\left(e_{2} y\right)=e_{2} x e_{2} y$. Then $\sigma \in \operatorname{Hom}_{R}\left(e_{2} R, e_{2} R\right)$, and since $e_{2} x e_{2}$ is nilpotent, $\sigma$ is not a monomorphism. So ker $\sigma \neq(0)$. Since $\operatorname{Hom}_{R}\left(e_{2} R, e_{1} R\right) \neq 0$, there exists an embedding $\eta: e_{2} R \rightarrow e_{1} R$. Now $\eta \sigma: e_{2} R \rightarrow e_{1} R$, and since $\operatorname{ker} \sigma \neq(0)$, ker $\eta \sigma \neq(0)$. By Lemma 5, $\eta \sigma=0$. Since $\eta$ is a monomorphism, we have $\sigma=0$. Thus $e_{2} x e_{2}=0$, and $e_{2} \mathrm{Ne}_{2}=0$. So $e_{2} R e_{2}$ is a division ring. Further $e_{2} R e_{2}=e_{2} R$ since $e_{2} R e_{1}=(0)$. Thus $e_{2} N=0$, and $e_{2} R$ is a minimal right ideal. Now $e_{1} R$ is uniform because it is quasi-injective and indecomposable. Since $0 \neq e_{1} R e_{2} R$ is the sum of the images of all $R$-homomorphisms of $e_{2} R$ into $e_{1} R$, the fact that $e_{2} R$ is minimal and $e_{1} R$ is uniform yields that $e_{1} R e_{2} R$ itself is the unique minimal right subideal of $e_{1} R$, is isomorphic to $e_{2} R$, and is contained in every nonzero right subideal of $e_{1} R$. We claim that $e_{1} N e_{1}=0$. Let $0 \neq e_{1} x e_{1} \in e_{1} N e_{1}$. Since $N$ is nil, $e_{1} x e_{1}$ is nilpotent. Then $\sigma: e_{1} R \rightarrow e_{1} R$ defined by $\sigma\left(e_{1} r\right)=e_{1} x e_{1} r$ is an endo- 
morphism of $e_{1} R$ with $\operatorname{ker} \sigma \neq(0)$. Let $A=\operatorname{ker} \sigma$. Then $e_{1} R e_{2} R \subset A$, and we have $e_{1} x e_{1} R e_{2}=(0)$. On the other hand, $e_{1} R e_{2} R \cong e_{1} x e_{1} R$ yields that $e_{1} x e_{1} R e_{2} \neq(0)$. This is a contradiction. Hence $e_{1} N e_{1}=(0)$, and $e_{1} R e_{1}$ is a division ring. Now using the fact that $\operatorname{Hom}_{R}\left(e_{1} R, e_{1} R\right)$ is a division ring and that $e_{1} R$ is quasi-injective, it follows that every member of $\operatorname{Hom}\left(e_{1} R e_{2} R, e_{1} R e_{2} R\right)$ admits a unique extension to an endomorphism of $e_{1} R$. Further, every endomorphism of $e_{1} R$ maps $e_{1} R e_{2} R$ into itself since $\mathrm{e}_{1} R e_{2} R$ is the unique minimal subideal of $e_{1} R$. Thus $\operatorname{Hom}\left(e_{1} R e_{2} R, e_{1} R e_{2} R\right) \cong \operatorname{Hom}\left(e_{1} R, e_{1} R\right)$. Since $e_{1} R e_{2} R \cong e_{2} R$, we obtain $e_{1} R e_{1} \cong e_{2} R e_{2}$.

Now $e_{1} N=e_{1} N e_{2}$ because $e_{1} N e_{1}=(0)$. Since $e_{1} R e_{2} R \cong e_{1} N$, we get $e_{1} N=e_{1} R e_{2}=e_{1} R e_{2} R$. Thus $M=e_{1} R e_{2}$ is a one-dimensional right vector space over $D=e_{2} R e_{2}$. We show that $M$ is also a one-dimensional left $e_{1} R e_{1}$-space. Let $X=\left(\begin{array}{cc}e_{1} R e_{1} & M \\ 0 & 0\end{array}\right) \cong R / A$, where $A=\left(\begin{array}{ll}0 & 0 \\ 0 & D\end{array}\right)$. Then $X$ is quasi-injective. Let $0 \neq x \in M$, and let $y \in M$. Consider $\sigma:\left(\begin{array}{cc}0 & M \\ 0 & 0\end{array}\right) \rightarrow\left(\begin{array}{cc}0 & M \\ 0 & 0\end{array}\right)$ defined by $\sigma\left(\begin{array}{cc}0 & x c \\ 0 & 0\end{array}\right)=\left(\begin{array}{cc}0 & y c \\ 0 & 0\end{array}\right)$, for $c \in D$. Then $\sigma$ is an $R$-endomorphism, so it can be extended to an endomorphism $\eta$ of $X$. Let $\eta\left(\begin{array}{ll}e_{1} & 0 \\ 0 & 0\end{array}\right)=\left(\begin{array}{ll}a & b \\ 0 & 0\end{array}\right)$. Then we have $\left(\begin{array}{ll}0 & y \\ 0 & 0\end{array}\right)=\sigma\left(\begin{array}{ll}0 & x \\ 0 & 0\end{array}\right)=\eta\left(\begin{array}{ll}0 & x \\ 0 & 0\end{array}\right)=$ $\left(\begin{array}{cc}0 & a x \\ 0 & 0\end{array}\right)$. Thus $y=a x$, so $M=e_{1} R e_{1} x$. So $M$ is a one-dimensional left vector space over $e_{1} R e_{1}$. Thus, for each $d \in e_{1} R e_{1}$, there exists a unique $d^{\prime} \in e_{2} R e_{2}$ such that $d x=x d^{\prime}$. Define $\theta: e_{1} R e_{1} \rightarrow e_{2} R e_{2}$ by $\theta(d)=d^{\prime}$. Then $\theta$ is an isomorphism, and we may identify $d$ and $d^{\prime}$. Then $\eta:\left(\begin{array}{ll}D & D \\ 0 & D\end{array}\right) \rightarrow\left(\begin{array}{cc}D & M \\ 0 & D\end{array}\right)$ defined by $\eta\left(\begin{array}{ll}a & b \\ 0 & c\end{array}\right)=\left(\begin{array}{ll}a & b x \\ 0 & c\end{array}\right)$ is an isomorphism.

Conversely, if $R$ satisfies (i), then, by Koehler [13], $R$ is a $Q C$ ring, hence a PCQI-ring. If $R$ satisfies (ii), then straightforward computation shows that $R$ is a right $P C Q I$-ring.

Since every right $Q C$-ring is a left $Q C$-ring and $\left(\begin{array}{ll}D & D \\ 0 & D\end{array}\right)$ is also a left $P C Q I$-ring, we get the following corollary.

CoROLlary. A nonlocal semiperfect right PCQI-ring is also a left PCQI-ring.

5. Local PCQI-rings. Theorem 13 and Theorems 14,15 , and 16 which follow generalize Klatt and Levy's [11] theorems for commutative pre-self-injective rings which are not domains. Throughout this section $M$ will denote the unique maximal right ideal of a local ring $R$. $M$ is then the Jacobson radical of $R$, and $R / M$ is a division ring.

THEOREM 14. Let $R$ be a local right PCQI-ring with maximal ideal $M$. Then either $R$ is a right valuation ring or $M^{2}=(0)$ and $M_{R}$ has composition length 2. 
Proof. First note that for all nonzero right ideals $A, R / A$ is indecomposable quasi-injective and hence uniform. Now we show that all nonzero right ideals are either minimal or essential. Let $A, B$ be nonzero right ideals such that $A \cap B=(0)$. We claim that $A$ is minimal. Let $C$ be a nonzero right ideal properly contained in $A$. Then $R / C$ is quasi-injective and not uniform since $A / C \cap$ $(B+C) / C=0$. This is a contradiction, so $A$ is minimal. Similarly, $B$ is minimal. In particular, it follows that any maximal independent family of minimal right ideals can contain at most two members.

If Soc $R_{R}=(0)$, then all nonzero right ideals are essential. Let $A, B$ be two nonzero right ideals. If neither $A \varsubsetneqq B$ nor $B \varsubsetneqq A$, then $R / A \cap B$ is quasi-injective but not uniform since $A /(A \cap B) \cap B /(A \cap B)$ $=(\overline{0})$. As before, this is a contradiction. So either $A \subseteq B$ or $B \subseteq A$.

If Soc $R_{R}$ consists of a unique minimal right ideal then it is clear that $R$ is a right valuation ring.

Finally, suppose Soc $R_{R}=A \oplus B$, where $A, B$ are minimal right ideals. Then $R$ cannot be prime. Let $x \in M$, and consider $x R$. If $x R$ is not minimal, then $x R$ is quasi-injective and decomposable. Then $x R=A \oplus B$. In any case, for all $x \in M, x \in \operatorname{Soc} R_{R}$. This implies that $M^{2}=(0)$, and the composition length of $M$ is 2 , completing the proof.

The next two theorems give the structure of non-prime local right PCQI-rings. Prime local PCQI-rings are discussed in the next section.

THEOREM 15. For a nonprime right valuation ring $R$, the following are equivalent:

(i) $R$ is a right PCQI-ring.

(ii) $R$ is a right duo almost maximal valuation ring of rank 0 such that any left ideal containing a nonzero right ideal is twosided.

Proof. (i) $\Rightarrow$ (ii). Since $R$ is not prime, $M$ is nil by Proposition 3. So, if $x R$ is a nontrivial principal right ideal of $R, x R$ is quasiinjective. Since $x R$ is essential in $R$, the injective hull of $x R$ is the same as that of $R$. Hence, by Johnson and Wong [10], $R x R \subseteq x R$. So $x R$ is a two-sided ideal of $R$. Thus $R$ is a right duo ring. Since each proper homomorphic image of a PCQI-ring is a $Q C$-ring, the proof of (i) $\Rightarrow$ (ii) as well as that of (ii) $\Rightarrow$ (i) is completed by a theorem of Koehler [13].

THEOREM 16. For a local ring $R$ with $M^{2}=(0)$ and the composition length of $M_{R}$ equal to 2, the following are equivalent: 
(i) $R$ is a right PCQI-ring.

(ii) For each nonzero right ideal $A$ in $R$ and for each $m_{1}, m_{2} \notin A$, the congruence $x m_{1} \equiv m_{2}(\bmod A)$ has a solution, $x=\alpha$, such that $\alpha A \subset A$.

Proof. Under the hypothesis the only nonzero right ideals $A$ of $R$ different from $M$ and $R$ are minimal right ideals, and $M / A$ is a simple right $R$-module.

(i) $\Rightarrow$ (2) Let $A$ be a nontrivial right ideal in $R$, and let $m_{1}, m_{2} \in R$ such that $m_{1}, m_{2} \notin A$. Then $\bar{m}_{1} R=M / A=\bar{m}_{2} R$, and the mapping $\sigma: M / A \rightarrow M / A$ which sends $\bar{m}_{1} r$ to $\bar{m}_{2} r$ is a well-defined $R$-homomorphism. Since $R / A$ is quasi-injective, $\sigma$ can be lifted to $\sigma^{*} \in \operatorname{Hom}_{R}(R / A, R / A)$. Let $\sigma^{*}(\overline{1})=\bar{\alpha}$. Then $\bar{\alpha} m_{1}=\bar{m}_{2}$. Hence $x m_{1} \equiv m_{2}(\bmod A)$ has a solution $x=\alpha$. Clearly $\alpha A \subset A$.

(ii) $\Rightarrow$ (i) We only need to prove that if $A$ is a nontrivial right ideal of $R$ and $\sigma: M / A \rightarrow R / A$, is a nonzero $R$-homomorphism, then $\sigma$ can be extended to an $R$-homomorphism $\sigma^{*}: R / A \rightarrow R / A$. Let $m \in M$, where $m \notin A$. Then $M / A=\bar{m} R$. Also, $\sigma(M / A)=M / A$. Let $\sigma(\bar{m})=\bar{m} r$. Since $M^{2}=(0), r \notin M$. So $r$ is invertible, and $m r \notin A$. Let $\alpha \in R$ be chosen such that $\alpha m \equiv \operatorname{mr}(\bmod A)$, and $\alpha A \subseteq A$. Then $\sigma^{*}(\bar{r})=\bar{\alpha} R$ is well-defined, and it extends $\sigma$, completing the proof.

The example which follows shows that a local right PCQI-ring is not necessarily a left $P C Q I$-ring.

ExAmple. Let $F$ be a field which has a monomorphism $\rho: F \rightarrow F$ such that $[F: \rho(F)]>2$. Take $x$ to be an indeterminate over $F$. Make $V=x F$ into a right vector space over $F$ in a natural way. Let $R=\{(\alpha, x \beta) \mid \alpha, \beta \in F\}$. Define

$$
\left(\alpha_{1}, x \beta_{1}\right)+\left(\alpha_{2}, x \beta_{2}\right)=\left(\alpha_{1}+\alpha_{1}, x \beta_{1}+x \beta_{2}\right)
$$

and

$$
\left(\alpha_{1}, x \beta_{1}\right)\left(\alpha_{2}, x \beta_{2}\right)=\left(\alpha_{1} \alpha_{2}, x\left(\rho\left(\alpha_{1}\right) \beta_{2}+\beta_{1} \alpha_{2}\right)\right) .
$$

Then $R$ is a local ring with identity with the maximal ideal

$$
M=\{(0, x \alpha) \mid \alpha \in F\} .
$$

In fact, $M$ is also a minimal right ideal and $M^{2}=(0)$. Thus $R$ is a right PCQI-ring. Further, if $\left\{\alpha_{i}\right\}_{i \in I}$ is a basis of $F$ as a vector space over $\rho(F)$ then straightforward computations yield that $M=\oplus \sum R\left(0, x \alpha_{1}\right)$ as a direct sum of irreducible left $R$-modules $R\left(0, x \alpha_{i}\right)$. Since card $I>2$, it follows by Theorem 14 that $R$ is not a left $P C Q I$-ring. 


\section{Prime local PCQI-rings.}

THEOREM 17. Let $R$ be a prime local right PCQI-ring. Then $R$ is a right valuation domain, hence right semihereditary.

Proof. By Theorem 14, $R$ is a right valuation ring. Let $A$ denote the intersection of all nonzero two-sided ideals of $R$. The proof that $R$ is a domain falls into three cases.

(i) $A=(0)$.

Let $x, y \in R$ such that $x y=0$. Suppose $y \neq 0$. Then $y R$ is a nonzero right ideal of $R$. Since $R$ is right valuation and $A=(0)$, $y R$ must contain a nonzero two-sided ideal of $R$. Further, each proper homomorphic image of $R$ is a local $Q C$-ring, hence a duo ring [13]. This implies that $y R$ is two-sided. Hence $x=0$, and $R$ is an integral domain.

(ii) $A \neq(0)$ and $A \neq M$.

Under these hypotheses, $A$ cannot be a prime ideal. So there exist $x, y \in R$ such that $x R y \subseteq A, x \notin A$ and $y \notin A$. Since $R$ is right valuation, $A \subseteq x R$ and $A \subseteq y R$. So both $x R$ and $y R$ are two-sided ideals. For definiteness, let $x R \subseteq y R$. Then $(x R)^{2} \subseteq(x R)(y R) \subseteq A R=A$ gives that $(x R)^{2}=A$ by the minimality of $A$. Also $A=A^{2}$, hence $(x R)^{2}=(x R)^{4}$. It follows that $x^{2} R=x^{4} R$. Then $x^{2}=x^{4} r$, for some $r \in R$, and $x^{2}\left(1-x^{2} r\right)=0$. So $x^{2}=0$. Thus $A=(0)$, and this case cannot occur.

(iii) $A=M$.

Let $S \subset R$, and let $r(S)$ denote the right annihilator of $S$ in $R$. Let $Z(R)=\{x \in R \mid r(x)$ is an essential right ideal $\}$. Then $Z(R)$ is an ideal in $R$ called the right singular ideal.

Since $R$ is a right valuation ring, $R$ is immediately a domain if $Z(R)=(0)$.

So assume that $Z(R) \neq(0)$. Then $Z(R)=M$, and each element in $M$ is a right zero divisor. So $x \in M$ implies that $x R$ is proper cyclic, hence quasi-injective. Also $x R$ is an essential right ideal in $R$. By Johnson and Wong [10], $R x R \subseteq x R$. Hence $x R$ is two-sided. So $R$ is a prime right duo ring, and it follows that $R$ is a domain.

7. PCQI-domains. In this section we discuss right $P C Q I$-rings which are integral domains and prove that these are right Öredomains. This generalizes the result of Faith [4]. Our proof, in this case, though it runs on the same lines as that of Faith, does not use Faith's result.

Proposition 18. Let $R$ be a right PCQI-domain, and let $I$ be a nonessential right ideal of $R$. Then $R / I$ is an injective right $R$ - 
module containing a copy of $R$.

Proof. Since $I$ is nonessential, there exists a nonzero right ideal $J$ in $R$ such that $I \cap J=0$. Let $a \in J$ such that $a \neq 0$. Then $a R \cap I \leqq J \cap I=0 . \quad$ Consider $\quad r(a+I)=\{x \in R \mid a x \in I\} . \quad$ Clearly $r(a+I)=0$. So $R / I$ contains a copy of $R$. Since $R / I$ is also quasiinjective, this implies that $R / I$ is injective by [17].

For a right $R$-module $A$, let $\hat{A}$ denote the injective hull of $A$.

Proposition 19. Let $R$ be a right PCQI-domain which is not a right Öre-domain. Then $\hat{R}$ is finitely presented.

Proof. Let $a \in R$ such that $a \neq 0$ and $a R$ is not essential. Then $R / a R$ is injective. Since $R / a R$ contains a copy of $R$ and is injective, $R / a R$ contains a copy of $\hat{R}$. Then $R / a R=Y / a R \oplus X / a R$, where $X / a R \cong \hat{R}$. Now $Y / a R$ is cyclic. So $Y=a R+b R$, for some $b \in R$, and the short exact sequence $0 \rightarrow Y \rightarrow R \rightarrow R / Y \cong X / a R \cong \hat{R} \rightarrow 0$ shows that $\hat{R}$ is finitely presented.

THEOREM 20. A right PCQI-domain $R$ is a right Öre-domain.

Proof. Let $R$ be a right PCQI-domain. Suppose $R$ is not a right Ore-domain. Then, as in Proposition 19, there exists $a \in R$ such that $R / a R=Y / a R \oplus X / a R$, where $X / a R \cong \hat{R} \cong R / Y$ and $Y=a R+b R$. We also get that $R=X+Y$, where $X \cap Y=a R$. This yields an exact sequence $0 \rightarrow a R \rightarrow X \times Y \rightarrow R \rightarrow 0$ which splits. So $X \times Y \cong$ $a R \times R \cong R \times R$. This implies that $Y=a R+b R$ is a finitely generated projective right ideal. Since $\hat{R} \cong R / Y, 0 \rightarrow Y \rightarrow R \rightarrow \hat{R} \rightarrow 0$ is exact. Then $Y \bigotimes_{R} \hat{R} \rightarrow R \bigotimes_{R} \hat{R} \rightarrow \hat{R} \bigotimes_{R} \hat{R} \rightarrow 0$ is exact. Also, a finitely generated projective $R$-module is essentially finitely related. So, by Cateforis ([3], Proposition 1.7), $(a R+b R) \bigotimes_{R} \hat{R}$ is projective as an $\hat{R}$-module. Then $Y \bigotimes_{R} \hat{R}$ is a direct summand of a free $\hat{R}$ module. Now $Z\left(\hat{R}_{\hat{R}}\right)=0$, hence $Z\left(Y \bigotimes_{R} \hat{R}\right)=0$ because $Y \bigotimes_{R} \hat{R}$ is a direct summand of a free $\hat{R}$-module. Now consider $Y \otimes_{R} \hat{R} \stackrel{i}{\rightarrow}$ $R \bigotimes_{R} \hat{R} \rightarrow \hat{R} \bigotimes_{R} \hat{R} \rightarrow 0$. Again, by Cateforis ([3], Lemma 1.8), $\operatorname{ker} i=Z\left(Y \bigotimes_{R} \hat{R}\right)=0$. So $0 \rightarrow Y \bigotimes_{R} \hat{R} \stackrel{i}{\rightarrow} R \bigotimes_{R} \hat{R} \rightarrow \hat{R} \bigotimes_{R} \hat{R} \rightarrow 0$ is exact. Since $R \bigotimes_{R} \hat{R} \cong \hat{R}$, let $f: R \bigotimes_{R} \hat{R} \rightarrow \hat{R}$ be the canonical isomorphism. Then fi: $Y \otimes_{R} \hat{R} \rightarrow \hat{R}$ is a monomorphism, and $Y \bigotimes_{R} \hat{R} \cong Y \hat{R}$. Since $Y$ is finitely generated, $Y \hat{R}$ is a finitely generated right ideal of $\hat{R}$. So $Y \hat{R}=e \hat{R}$, where $e^{2}=e$. Thus we have the following exact sequence: $\quad 0 \rightarrow e \hat{R} \rightarrow \hat{R} \rightarrow \hat{R} \bigotimes_{R} \hat{R} \rightarrow 0$, and $\hat{R} \bigotimes_{R} \hat{R} \cong \hat{R} / e \hat{R}=$ $(1-e) \hat{R}$. Hence $\hat{R} \otimes_{R} \hat{R}$ is isomorphic to a direct summand of $\hat{R}$. Since $Z\left(\hat{K}_{R}\right)=0, Z\left(\hat{R} \otimes_{R} \hat{R}\right)=0$. Since $\hat{R}=x R$, for some $x \in \hat{R}$, the 
kernel of the canonical map $f: \hat{R} \otimes_{R} \hat{R} \rightarrow \hat{R}$ defined by $f(a \otimes b)=a b$ is contained in $Z\left(\hat{R} \otimes_{R} \hat{R}\right)$ and hence must be zero. Since $f$ is surjective, $f$ is an isomorphism. By Silver ([18], Proposition 1.1), there exists an epimorphism in the category of rings from $R$ to $\hat{R}$.

Let $M$ be a right $\hat{R}$-module which is quasi-injective as a right $R$-module. We claim that $M$ is quasi-injective as a right $\hat{R}$-module. Let $0 \rightarrow A_{\hat{R}} \rightarrow M_{\hat{R}} \rightarrow B_{\hat{R}} \rightarrow 0$ be exact. Consider $0 \rightarrow \operatorname{Hom}_{\hat{R}}\left(B_{\hat{R}}, M_{\hat{R}}\right) \rightarrow$ $\operatorname{Hom}_{\hat{R}}\left(M_{\hat{R}}, M_{\hat{R}}\right) \rightarrow \operatorname{Hom}_{\hat{R}}\left(A_{\hat{R}}, M_{\hat{R}}\right)$. By Silver ([18], Corollary 1.3), $\operatorname{Hom}_{\hat{R}}\left(N, N^{1}\right) \cong \operatorname{Hom}_{R}\left(N, N^{1}\right)$, where $N, N^{1}$ are right $\hat{R}$-modules. Also $0 \rightarrow \operatorname{Hom}_{R}(B, M) \rightarrow \operatorname{Hom}_{R}(M, M) \rightarrow \operatorname{Hom}_{R}(A, M) \rightarrow 0$ is exact since $M_{R}$ is quasi-injective. Thus $0 \rightarrow \operatorname{Hom}_{\hat{R}}(B, M) \rightarrow \operatorname{Hom}_{\hat{R}}(M, M) \rightarrow$ $\operatorname{Hom}_{R}(A, M) \rightarrow 0$ is exact. So $M_{\hat{R}}$ is quasi-injective. Let $K$ be a cyclic right $R$-module. Then $K$ is a cyclic right $R$-module. Since $R$ is a right $P C Q I$-domain, $K_{R}$ is quasi-injective. Thus $K_{\hat{R}}$ is quasiinjective. Since $\hat{R}$ is right self-injective, $\hat{R}$ is a $Q C$-ring. So $\hat{R}$ is semiperfect and simple, hence simple artinian. Thus $\hat{R}$ is a division ring. This proves that $R$ is a right Öre-domain.

We conclude by a remark that we have not studied arbitrary prime right $P C Q I$-rings. This case remains open. Indeed, a characterization of right $P C Q I$-domains has not yet been obtained.

\section{REFERENCES}

1. J. Ahsan, Rings all of whose cyclic modules are quasi-injective, Proc. London Math. Soc., 27 (1973), 425-439.

2. R. Bumby, Modules which are isomorphic to submodules of each other, Archiv. der Math., 16 (1965), 184-185.

3. V. C. Cateforis, Flat regular quotient rings, Trans. Amer. Math. Soc., 138 (1969), 241-249.

4. C. Faith. When are proper cyclics injective, Pacific J. Math., 45 (1973), 97-112.

5 . - Lectures on Injective Modules and Quotient Rings, Springer-Verlag, Berlin, 1967.

6. M. Harada, Note on Quasi-injective modules, Osaka J. Math., 2 (1965), 351-356.

7. D. A. Hill, Semi-perfect q-rings, Math. Ann., 200 (1973), 113-121.

8. G. Ivanov, Non-local rings whose ideals are all quasi-injective, Bull. Australian Math. Soc., 6 (1972), 45-52.

9. S. K. Jain, S. Mohamed and Surjeet Singh, Rings in which every right ideal is quasi-injective, Pacific J. Math., 31 (1969), 73-79.

10. R. E. Johnson and E. T. Wong, Quasi-injective modules and irreducible rings, J. London Math. Soc., 36 (1961), 260-268.

11. G. B. Klatt and L. S. Levy, Pre-self-injective rings, Trans. Amer. Math. Soc., 137 (1969), 407-419.

12. A. Koehler, Rings for which every cyclic module is quasi-projective, Math. Ann., 189 (1970), 311-316.

13. - Rings with quasi-injective cyclic modules, Quar. J. Math. Oxford (2), 25 (1974), 51-55.

14. L. S. Levy, Commutative rings whose homomorphic images are self-injective, Pacific J. Math. 18 (1966), 149-153. 
15. S. Mohamed, q-rings with chain conditions, J. London Math. Soc., 2 (1970), 453-460. 16. B. L. Osofsky, Noncyclic injective modules, Proc. Amer. Math. Soc., 19 (1968), 1383-1384.

17. E. deRobert, Projectifs et injectifs relatifs, C. R. Acad. Sci. Paris, Ser. A, 286 (1969), 361-364.

18. L. Silver, Noncommutative localizations and applications, J. Algebra, 7 (1967), 44-76.

Received November 19, 1976.

Ohio University, Athens, OHio

Present address: Surjeet Singh, Guru Nanak Dev University, Amritsar, India.

Present address: Robin Symonds, Indiana University at Kokomo, Kokomo, Indiana 46901. 


\section{PACIFIC JOURNAL OF MATHEMATICS}

\section{EDITORS}

RICHARD ARENS (Managing Editor)

University of California

Los Angeles, California 90024

\section{J. DugunduI}

Department of Mathematics University of Southern California Los Angeles, California 90007

D. Gilbarg and J. Milgram

Stanford University

Stanford, California 94305

\author{
R. A. Beaumont \\ University of Washington \\ Seattle, Washington 98105
}

\section{ASSOCIATE EDITORS}
B. H. NeUmanN
F. WOLF
K. YosHIDA

\section{SUPPORTING INSTITUTIONS}

\author{
UNIVERSITY OF BRITISH COLUMBIA \\ CALIFORNIA INSTITUTE OF TECHNOLOGY \\ UNIVERSITY OF CALIFORNIA \\ MONTANA STATE UNIVERSITY \\ UNIVERSITY OF NEVADA \\ NEW MEXICO STATE UNIVERSITY \\ OREGON STATE UNIVERSITY \\ UNIVERSITY OF OREGON \\ OSAKA UNIVERSITY
}

\author{
UNIVERSITY OF SOUTHERN CALIFORNIA \\ STANFORD UNIVERSITY \\ UNIVERSITY OF HAWAII \\ UNIVERSITY OF TOKYO \\ UNIVERSITY OF UTAH \\ WASHINGTON STATE UNIVERSITY \\ UNIVERSITY OF WASHINGTON \\ AMERICAN MATHEMATICAL SOCIETY
}

The Supporting Institutions listed above contribute to the cost of publication of this Journal, but they are not owners or publishers and have no responsibility for its content or policies.

Mathematical papers intended for publication in the Pacific Journal of Mathematics should be in typed form or offset-reproduced, (not dittoed), double spaced with large margins. Please do not use built up fractions in the text of your manuscript. You may however, use them in the displayed equations. Underline Greek letters in red, German in green, and script in blue. The first paragraph or two must be capable of being used separately as a synopsis of the entire paper. Items of the bibliography should not be cited there unless absolutely necessary, in which case they must be identified by author and Journal, rather than by item number. Manuscripts, in triplicate, may be sent to any one of the editors. Please classify according to the scheme of Math. Reviews, Index to Vol. 39. All other communications should be addressed to the managing editor, or Elaine Barth, University of California, Los Angeles, California, 90024.

The Pacific Journal of Mathematics expects the author's institution to pay page charges, and reserves the right to delay publication for nonpayment of charges in case of financial emergency.

100 reprints are provided free for each article, only if page charges have been substantially paid. Additional copies may be obtained at cost in multiples of 50 .

The Pacific Journal of Mathematics is issued monthly as of January 1966. Regular subscription rate: $\$ 72.00$ a year (6 Vols., 12 issues). Special rate: $\$ 36.00$ a year to individual members of supporting institutions.

Subscriptions, orders for back numbers, and changes of address should be sent to Pacific Journal of Mathematics, 103 Highland Boulevard, Berkeley, California, 94708.

PUBLISHED BY PACIFIC JOURNAL OF MATHEMATICS, A NON-PROFIT CORPORATION

Printed at Kokusai Bunken Insatsusha (International Academic Printing Co., Ltd.), 8-8, 3-chome, Takadanobaba, Shinjuku-ku, Tokyo 160, Japan. 


\section{Pacific Journal of Mathematics}

Patricia Andresen and Marvin David Marcus, Weyl's inequality and

quadratic forms on the Grassmannian .......................

George Bachman and Alan Sultan, Regular lattice measures: mappings and

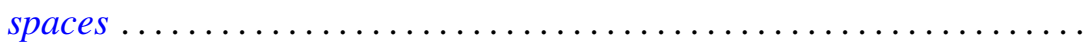

David Geoffrey Cantor, On certain algebraic integers and approximation by rational functions with integral coefficients ...................

James Richard Choike, On the value distribution of functions meromorphic in the unit disk with a spiral asymptotic value ..................

David Earl Dobbs, Divided rings and going-down................ 353

Mark Finkelstein and Robert James Whitley, Integrals of continuous

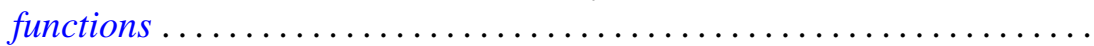

Ronald Owen Fulp and Joe Alton Marlin, Integrals of foliations on manifolds with a generalized symplectic structure ...............

Cheong Seng Hoo, Principal and induced fibrations .................

Wu-Chung Hsiang and Richard W. Sharpe, Parametrized surgery and

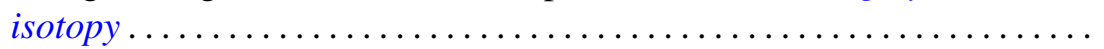

Surender Kumar Jain, Surjeet Singh and Robin Gregory Symonds, Rings whose proper cyclic modules are quasi-injective .................

Pushpa Juneja, On extreme points of the joint numerical range of commuting normal operators...

Athanassios G. Kartsatos, Nth order oscillations with middle terms of order $N-2$.

John Keith Luedeman, The generalized translational hull of a

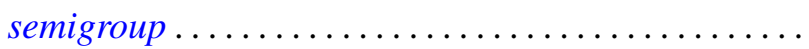

Louis Jackson Ratliff, Jr., The altitude formula and DVR's ...

Ralph Gordon Stanton, C. Sudler and Hugh C. Williams, An upper bound for the period of the simple continued fraction for $\sqrt{D}$...

David Westreich, Global analysis and periodic solutions of second order systems of nonlinear differential equations...

David Lee Armacost, Correction to: "Compactly cogenerated LCA

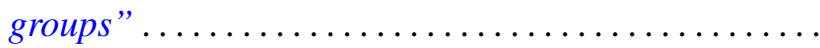

Jerry Malzan, Corrections to: "On groups with a single involution" .

David Westreich, Correction to: "Bifurcation of operator equations with unbounded linearized part" ...................... 